\title{
ON WEIERSTRASS SEMIGROUPS AT ONE AND TWO POINTS AND THEIR CORRESPONDING POINCARÉ SERIES
}

\author{
JULIO JOSÉ MOYANO-FERNÁNDEZ
}

\begin{abstract}
The aim of this paper is to introduce and investigate the Poincaré series associated with the Weierstraß semigroup of one and two rational points at a (not necessarily irreducible) nonsingular projective algebraic curve defined over a finite field, as well as to describe their functional equations in the case of an affine complete intersection.
\end{abstract}

\section{INTRODUCTION}

The origin of the Weierstraß semigroup lies in the "Lückensatz" pointed out by Karl Weierstraß in some of his lectures in the 1860's: For every point $P$ on a compact Riemann surface $X$ of genus $g$, there are exactly $g$ integers $l_{i}(P)$ with

$$
1=l_{1}(P)<\ldots<l_{g}(P)=2 g-1
$$

so that there is no meromorphic function on $X$ having a pole at $P$ of multiplicity $l_{i}(P)$ as its only singularity (see [19, III, pp. 297-307]). The set $G(P):=\left\{l_{i}(P)\right\}$ is called the gap set of $P$. After some remarkable results of Brill and Noether ([2]), Hurwitz realised that, if $\alpha, \beta$ belong to $\mathbb{N} \backslash G(P)$ and $z_{\alpha}, z_{\beta}$ are two functions having a pole of order $\alpha$ resp. $\beta$ at $P$ as their only singularities, then the function $z_{\alpha} z_{\beta}$ has a pole of order $\alpha+\beta$ at $P$ as its only singularity (cf. [5]). It implies that $\mathbb{N} \backslash G(P)$ is a semigroup (cf. 9], p. 409 after equation (6)): The Weierstraß semigroup associated with the point $P$.

1991 Mathematics Subject Classification. Primary 14H55; Secondary 13D40.

Key words and phrases. Weierstrass semigroup and discrete Manis valuation and Poincaré series.

Supported partially by the grant of the Spanish Government "Ministerio de Educación MTM2007-64704", in cooperation with the European Union in the framework of the founds "FEDER", by the grant of the Deutsches Akademischen Austauschdienst (DAAD)-La Caixa, and by the Deutsche Forschungsgemeinschaft (DFG). 
Let $X$ be a non-singular projective algebraic curve defined over a finite field $\mathbb{F}$. Let $P_{1}, \ldots, P_{r}$ be a set of rational points on $X$ and consider the family of (finite dimensional) $\mathbb{F}$-vector subspaces

$$
\Gamma(X, \underline{m P}):=\Gamma\left(X, m_{1} P_{1}+\ldots+m_{r} P_{r}\right)
$$

with $m_{i} \in \mathbb{Z}$ for all $i=1, \ldots, r$ (cf. Subsect. 2.2 below). This family gives rise to a $\mathbb{Z}^{r}$-multi-index filtration on the $\mathbb{F}$-algebra $A:=$ $\bigcup_{m \in \mathbb{Z}^{r}} \Gamma(X, \underline{m P})$ of the affine curve $X \backslash\left\{P_{1}, \ldots, P_{r}\right\}$. This multiindex filtration is related to Weierstraß semigroups (with respect to several points in general, see [7]) and, in the case of finite fields, to the methodology for trying to improve the Goppa estimation of the minimal distance of algebraic-geometrical codes (see [4]). Furthermore, a connection of that filtration with global geometrical-topological aspects in a particular case is shown in [3].

Poincaré series are typically series for which the coefficients represent discrete information about the class of the study objects - the Weierstraß semigroup in this case. Thus, a natural question, which this work is devoted to, is to define in a proper way the notion of Poincaré series and to describe it, in the case of a Weierstraß semigroup associated with one or two points. This will be made by associating such a series to the dimensions of the vector spaces $\Gamma(X, \underline{m P})$.

The article goes as follows. Sect. 2 is devoted to the definition and main properties of the Weierstraß semigroup for not necessarily irreducible curves using the notion of Manis valuation. In Sect. 3 we introduce and describe the Poincaré series of the Weierstraß semigroup associated with one and two points. A formula for the conductor of the semigroup for the case $X$ to be non-singular in the affine part, as well as descriptions for the genus of $X$ and the gaps of the semigroup if the curve is affine complete intersection are provided. Finally we consider symmetry properties in Sect. 4 (revising the work of Delgado [7]), from which we deduce functional equations for the Poincaré series.

\section{WeIERSTRASS SEMIGROUP FOR REDUCIBLE CURVES}

2.1. Reducible curves and Manis valuations. We can extend the concept of valuation to start from a ring satisfying certain properties, instead of a field. More precisely, let $\mathcal{K}$ be a ring with large Jacobson radical (i.e., a ring in which every prime ideal containing the Jacobson radical is maximal; for instance, a semilocal ring) and being its own total ring of fractions. A surjective map $w: \mathcal{K} \rightarrow \mathbb{Z}_{\infty}:=\mathbb{Z} \cup\{\infty\}$ such that $w(1)=0, w(0)=\infty, w(a b)=w(a)+w(b)$, for all $a, b \in \mathcal{K}$, and 
$w(a+b) \geq \min \{w(a), w(b)\}$, for all $a, b \in \mathcal{K}$, is called a Manis valuation of the ring $\mathcal{K}$ (cf. [10, Chapter I, Def. 2.8, p. 9]).

Let $X$ be a reducible projective curve of arithmetical genus $g_{X}$ defined over a finite field $\mathbb{F}$, which is locally a complete intersection (or simply a curve from now on). Let $X=X_{1} \cup \ldots \cup X_{r}$ be its decomposition into irreducible components. A function field $K\left(X_{i}\right)$ can be associated to each $X_{i}$, and $K(X):=K\left(X_{1}\right) \times \ldots \times K\left(X_{r}\right)$ is the ring of rational functions over $X$. A point of $X$ is called regular if it lies on just one irreducible component of $X$ and if it is a simple point of this component. Let us take $P$ a regular point of $X$. Then there exists some $\kappa \in\{1, \ldots, r\}$ such that $P \in X_{\kappa}$ and the local ring of $X$ at $P$ coincides with the local ring of $X_{\kappa}$ at $P$, and therefore, one can associate with $P$ a discrete valuation $v_{P}: K\left(X_{\kappa}\right) \rightarrow \mathbb{Z}_{\infty}$. Let us consider $z=\left(z_{1}, \ldots, z_{r}\right) \in K(X)$. For every $1 \leq j \leq r$, we define

$$
w_{P}^{\prime}\left(z_{j}\right)= \begin{cases}v_{P}\left(z_{j}\right), & \text { if } j=\kappa ; \\ \infty, & \text { otherwise. }\end{cases}
$$

It is easy to check that the map $w_{P}: K(X) \rightarrow \mathbb{Z}_{\infty}, z \mapsto \min _{1 \leq j \leq r} w_{P}^{\prime}\left(z_{j}\right)$ is a discrete Manis valuation of the ring $K(X)$.

Let $\underline{P}:=\left\{P_{1}, \ldots, P_{r}\right\}$ be a set of non-singular points on $X$, and set $w_{i}=w_{P_{i}}$ a discrete Manis valuation of $K(X)$ associated with each $P_{i}$, for all $1 \leq i \leq r$. If we consider the affine coordinate ring $\Gamma\left(X^{\prime}, \mathcal{O}_{X}\right)$ of the affine curve $X^{\prime}:=X \backslash \underline{P}$, then we may define the Weierstraß semigroup of $X$ at $\underline{P}$ as the following sub-semigroup of $\left(\mathbb{Z}_{\infty}\right)^{r}$ :

$$
\Gamma_{\underline{P}}:=\left\{-\left(w_{1}, \ldots, w_{r}\right)(f) \mid f \in \Gamma\left(X^{\prime}, \mathcal{O}_{X}\right)\right\} .
$$

Notations. Let $\underline{n}=\left(n_{1}, \ldots, n_{r}\right) \in \mathbb{Z}^{r}, J=\left\{i_{1}, \ldots, i_{d}\right\} \subseteq I:=$ $\{1, \ldots, r\}$. Consider the subsets

$$
\begin{gathered}
\bar{\nabla}_{J}(\underline{n})=\left\{\left(m_{1}, \ldots, m_{r}\right) \in \mathbb{Z}^{r} \mid m_{i}=n_{i} \forall i \in J, m_{j}<n_{j} \forall j \notin J\right\} \\
\bar{\nabla}(\underline{n})=\bigcup_{i=1}^{r} \bar{\nabla}_{i}(\underline{n}) ;
\end{gathered}
$$

and $\nabla_{J}(\underline{n}):=\bar{\nabla}_{J}(\underline{n}) \cap \Gamma_{\underline{P}}, \nabla(\underline{n}):=\bar{\nabla}(\underline{n}) \cap \Gamma_{\underline{P}}$, as well as $\nabla_{i}^{r}(\underline{n}):=$ $\bigcup_{i \in J} \nabla_{J}(\underline{n})$. The vectors of the standard basis of $\mathbb{Z}^{r}$ will be denoted by $e_{1}, \ldots, e_{r}$.

Definition 2.1. An element $\underline{n}=\left(n_{1}, \ldots, n_{r}\right)$ of the semigroup $\Gamma_{\underline{P}}$ is said to be maximal for $\Gamma_{\underline{P}}$ if $\nabla(\underline{n})=\varnothing$. If, moreover, $\nabla_{J}(\underline{n})=\varnothing$ for every $J \subsetneq I$, then the maximal element is called absolute maximal. A maximal element for $\Gamma_{\underline{P}}$ is called relative maximal if $\nabla_{J}(\underline{n}) \neq \varnothing$ for every $J \subsetneq I$ with $\sharp J \geq \overline{2}$ (and $\nabla_{J}(\underline{n})=\varnothing$ if $\sharp J=1$ ). 
Remark 2.2. For the case $r=2$, the concepts of relative and absolute maximal coincide. Thus we will simply say "maximal" points of $\Gamma_{P_{1}, P_{2}}$. The set of maximal points of $\Gamma_{P_{1}, P_{2}}$ will be denoted by $\mathcal{M}_{P_{1}, P_{2}}$.

2.2. The Riemann-Roch theorem for reducible curves. Let $X$ be a curve. Let $\mathcal{O}_{X}$ be the sheaf of local rings on $X$. We can take, for each invertible sheaf $\mathcal{L}$ on $X$, a divisor $D$ with support contained in the set of regular points of the curve such that $\mathcal{L}=\mathcal{L}(D)$, where $\mathcal{L}(D)$ is the divisorial sheaf related to $D$. As $\mathcal{L}(D)$ is coherent, the cohomology groups $H^{q}(X, \mathcal{L}(D))$ are $\mathbb{F}$-vector spaces of finite dimension (if $q \geq 2$ its dimension is 0$)$. Furthermore, since $\mathcal{L}(D) \cap \mathcal{O}_{X}$ is a coherent sheaf too, we can define the degree of the divisor $D$ as

$\operatorname{deg}(D)=\operatorname{dim}_{\mathbb{F}} \Gamma\left(X, \mathcal{L}(D) /\left(\mathcal{L}(D) \cap \mathcal{O}_{X}\right)\right)-\operatorname{dim}_{\mathbb{F}} \Gamma\left(X, \mathcal{O}_{X} /\left(\mathcal{L}(D) \cap \mathcal{O}_{X}\right)\right)$.

Such a definition extends in a natural way the classical one. Since $X$ is locally a complete intersection, the dualizing sheaf of $X$ is an invertible sheaf on $X$ and we can take the corresponding canonical divisor $K$ such that $\operatorname{supp}(K)$ is contained in the set of regular points on $X$. By setting

$$
\begin{aligned}
& \ell(D)=\operatorname{dim}_{\mathbb{F}} H^{0}(X, \mathcal{L}(D))=\operatorname{dim}_{\mathbb{F}} \Gamma(X, \mathcal{L}(D)) \\
& i(D)=\operatorname{dim}_{\mathbb{F}} H^{1}(X, \mathcal{L}(D))=\ell(D-K) \\
& \gamma=\operatorname{dim}_{\mathbb{F}} H^{0}\left(X, \mathcal{O}_{X}\right)=\text { number of connected parts of } X,
\end{aligned}
$$

the Riemann-Roch theorem for reducible curves can be proven (see [13, p. 103]):

Theorem 2.3 (Riemann-Roch). For every divisor $D$ on $X$, we have

$$
\ell(D)-i(D)=\operatorname{deg}(D)-g_{X}+\gamma \text {. }
$$

If $D=\left\{\left(U_{i}, f_{i}\right)\right\}_{i \in I}$ is a Cartier divisor in $X$, and $P$ is a regular rational point on $X$, we denote by $w_{P}(D)$ the value $w_{P}\left(f_{j}\right)$ if $P \in U_{j}$ (in the sense of Manis). Usual notations for effectivity of $D$ (namely $D>0$ ) and linear equivalence between two divisors $\left(D \sim D^{\prime}\right)$ hold.

Let us consider a set $\left\{P_{1}, \ldots, P_{r}\right\}$ of non-singular rational points on $X$, a vector $\underline{n}=\left(n_{1}, \ldots, n_{r}\right) \in \mathbb{Z}^{r}$ and the divisor $\underline{n P}=\sum_{i=1}^{r} n_{i} P_{i}$. Denote the $\mathbb{F}$-vector space of global sections of the invertible sheaf $\mathcal{L}(\underline{n P})$ by $\Gamma(X, \underline{n P})$, its dimension by $\ell(\underline{n P})$ and $\ell(K-\underline{n P}):=\operatorname{dim}_{\mathbb{F}} \Gamma(X, \mathcal{L}(K-$ $\underline{n P}))$. We have

$$
\begin{aligned}
\Gamma(X, \underline{n P}) & =\left\{f \in K(X)^{*} \mid(f)+\underline{n P}>0\right\} \\
& =\left\{f \in K(X)^{*} \mid w_{i}(P) \geq-n_{i}, i=1, \ldots, r\right\},
\end{aligned}
$$

where $K(X)^{*}:=K(X) \backslash\{0\}$. Notice that $\Gamma(X, \underline{n P}) \supseteq \Gamma(X, \underline{m P})$ for every $\underline{n}, \underline{m} \in \mathbb{Z}^{r}$ with $n_{i} \geq m_{i}$, for all $i \in\{1, \ldots, r\}$; hence the set $\{\Gamma(X, \underline{n} P)\}_{\underline{n}} \in \mathbb{Z}^{r}$ defines a filtration given by multi-indices (or multiindex filtration) of the curve. 
2.3. Main properties of the semigroup. We collect now some basic properties and definitions around the Weierstraß semigroup.

Definition 2.4. An element $\underline{m} \in \mathbb{Z}^{r}$ is said to be a gap of $\Gamma_{P}$ if $\underline{m} \notin \Gamma_{P}$. Otherwise $\underline{m}$ is said to be a non-gap of $\Gamma_{\underline{P}}$.

Let us mention two important properties of these concepts (their proofs, also valid for Manis valuations, can be found in [7]; or [4, Sect. 2]).

Lemma 2.5. Let $\underline{m} \in \mathbb{Z}^{r}$, then $\underline{m}$ is a non-gap of $\Gamma_{\underline{P}}$ if and only if for all $i \in\{1, \ldots, r\}$ one has $\ell(\underline{m P})=\ell\left(\left(\underline{m}-e_{i}\right) P\right)+\overline{1}$.

Lemma 2.6. If $X$ is a curve of genus $g$ and $\underline{m}=\left(m_{1}, \ldots, m_{r}\right) \in \mathbb{Z}^{r}$ is a gap, then $0 \leq m_{1}+\ldots+m_{r}<2 g_{X}$.

Definition 2.7. Let $\tau \in \mathbb{Z}_{>0}^{r}$. The Weierstraß semigroup $\Gamma_{\underline{P}}$ is said to be symmetric with respect to $\tau$ if it satisfies the following property (cf. [7]): "If $\underline{n} \in \mathbb{Z}^{r}$, then $\underline{n} \in \Gamma_{\underline{P}}$ if and only if $\nabla(\tau-\underline{n})=\varnothing "$.

Notice that for $r=1$ this means: The semigroup $\Gamma_{P}$ is symmetric if there exists an integer $n$ such that the mapping $\mathbb{Z} \rightarrow \mathbb{Z}$, given by $z \mapsto n-z$, maps elements of $\Gamma_{P}$ onto gaps and gaps onto elements of $\Gamma_{P}$ (cf. definition in [8, p. 32]). Furthermore, the case $r=1$ is remarkable in the following sense:

Lemma 2.8. Let $r=1$. Then $\Gamma_{P}$ is a numerical semigroup.

This implies that $\Gamma_{P}$ possesses a conductor, denoted by $c\left(\Gamma_{P}\right)$, when $r=1$ (see e. g. [15]). The gap $g\left(\Gamma_{P}\right):=c\left(\Gamma_{P}\right)-1$ is called the Frobenius number associated to the semigroup $\Gamma_{P}$.

Lemma 2.9. Let $X$ be a curve, $P_{1}, \ldots, P_{r}$ rational points on $X$ and $\Gamma_{P}$ the Weierstraß semigroup of $P_{1}, \ldots, P_{r}$. Let $\underline{m}:=\left(m_{1}, \ldots, m_{r}\right) \in \mathbb{Z}^{r}$.

(i) There exists a number $\vartheta \in \mathbb{Z} \backslash\{0\}$ so that $\vartheta\left(P_{i}-P_{j}\right)$ is a principal divisor, for any $i, j \in\{1, \ldots r\}$ with $i \neq j$.

(ii) For any $\lambda \in \mathbb{Z}$, then $\underline{m} \in \Gamma_{\underline{P}}$ if and only if $\underline{m}+\lambda(\vartheta,-\vartheta, 0, \ldots, 0) \in$ $\Gamma_{\underline{P}}$.

Proof. (i) Let $\operatorname{Pic}(X)$ be the set of isomorphism classes of invertible sheaves on $X$, and $\operatorname{Pic}^{0}(X)$ the subgroup of $\operatorname{Pic}(X)$ of those classes of degree 0 . The Jacobian variety of $X$ is isomorphic to $\operatorname{Pic}^{0}(X)$ (cf. [11, Chapter 7, Theorem 4.39]). Since the $\mathbb{F}$-points of $J$ are a finite group, then the divisor $P_{i}-P_{j} \in \operatorname{Pic}^{0}(X)$ is an element of finite order, for any $i, j \in\{1, \ldots, r\}, i \neq j$. (ii) is a consequence of (i).

We are in this article much more interested in the case $r=2$. Let us assume for the rest of this section $\Gamma_{\underline{P}}$ to be the Weierstraß semigroup of two rational points $P_{1}, P_{2}$ on $X$. 
Definition 2.10. Let us assume $\sharp(\mathbb{F})>2$. The smallest natural number $\vartheta \neq 0$ such that $\vartheta\left(P_{1}-P_{2}\right)$ is a principal divisor is called the period of the semigroup $\Gamma_{P_{1}, P_{2}}$.

Remark 2.11. Consider the discrete plane $\mathbb{Z}^{2}$. The period of the semigroup defines an important area on it, namely the parallelogram in $\Gamma_{P_{1}, P_{2}} \cap \mathbb{Z}^{2}$ determined by the vertices $(0,0),(0,2 g),(\vartheta, 2 g-\vartheta)$ and $(\vartheta,-\vartheta)$ not including the line joining $(0,0)$ and $(0,2 g)$, which will be called the fundamental corner of $\Gamma_{P_{1}, P_{2}}$, and will be denoted by $\mathcal{C}_{P_{1}, P_{2}}=\mathcal{C}$. By Lemma 2.9 (ii), any translation of $\mathcal{C}_{P_{1}, P_{2}}$ of vector $\lambda(\vartheta,-\vartheta)$ for every $\lambda \in \mathbb{Z} \backslash\{0\}$, reproduces the same distribution of points in $\Gamma_{P_{1}, P_{2}}$. Therefore, the points in $\mathcal{C}$ will play an essential role in the semigroup's knowledge, as we will see in the next sections, especially the set of maximal points of $\mathcal{C}$, i.e. the set $\mathcal{M}_{\mathcal{C}}:=\mathcal{M}_{P_{1}, P_{2}} \cap \mathcal{C}$.

\section{Poincaré series of the Weierstrass semigroups} ASSOCIATED WITH ONE AND TWO POINTS

This section is devoted to introduce the notion of Poincaré series associated with the multi-index filtration given by $\Gamma(X, \underline{m P})$ and to show its behaviour in the case of one and two points. For every $\underline{m} \in \mathbb{Z}^{r}$, set

$$
d(\underline{m}):=\operatorname{dim}_{\mathbb{F}} \frac{\Gamma(X, \underline{m P})}{\Gamma(X,(\underline{m}-\underline{1}) \underline{P})} .
$$

Definition 3.1. The Poincaré series of the Weierstraß semigroup $\Gamma_{\underline{P}}$ is defined to be

$$
P_{\Gamma_{\underline{P}}}(\underline{t}):=\sum_{\underline{m} \in \mathbb{Z}^{r}} d(\underline{m}) \underline{t} \underline{m},
$$

where $\underline{\underline{t}} \underline{\underline{m}}:=t_{1}^{m_{1}} \cdot \ldots \cdot t_{r}^{m_{r}}$.

The series $P_{\Gamma_{\underline{P}}}(\underline{t})$ contains negative powers of variables $t_{i}$, however it does not contain monomials $\underline{t} \underline{\underline{m}}$ with purely negative $\underline{m}$ (i.e., all components of which are negative). It will be convenient to consider $P_{\Gamma_{\underline{P}}}(\underline{t})$ as an element of the set of formal Laurent series in the variables $t_{1}, \ldots, t_{r}$ with integer coefficients without purely negative exponents; i.e., of expressions of the form $\sum_{\underline{m} \in \mathbb{Z}^{r} \backslash \mathbb{Z}_{\leq-1}^{r}} d(\underline{m}) \underline{t} \underline{m}$.

3.1. Poincaré series associated with one point. Consider $\Gamma_{P_{1}}=$ $\Gamma_{P}$ the Weierstraß semigroup associated with one rational point on $X$. In this case we have that $d(m)=1$ if and only if $m \in \Gamma_{P}$, hence $P_{\Gamma_{P}}(t)=\sum_{n \in \Gamma_{P}} t^{n}$.

Assume $X$ to be smooth on the affine part. Then $\Gamma_{P}$ is strictly generated by some elements $\left\{r_{0}, \ldots, r_{h}\right\}$ (cf. [1]). By setting

$$
\theta_{i}:=\operatorname{gcd}\left(r_{0}, \ldots, r_{i-1}\right)
$$


for $1 \leq i \leq h+1$ and $d_{i}:=\theta_{i} / \theta_{i+1}$ for $1 \leq i \leq h$, the semigroup $\Gamma_{P}$ satisfies the following property: Every element $n \in \Gamma_{P}$ can be written uniquely as

$$
n=\lambda_{0} r_{0}+\lambda_{1} r_{1}+\ldots+\lambda_{h} r_{h}
$$

with $\lambda_{0} \geq 0$ and $0 \leq \lambda_{i}<d_{i}$, for $1 \leq i \leq h$. This leads to the following description of the Poincaré series $P_{\Gamma_{P}}(t)$ (cf. [3, p. 1840]):

Proposition 3.2. Let $X$ be non-singular in the affine part. Let $\Gamma_{P}$ be the Weierstraß semigroup at a point $P$ on $X$. We have

$$
P_{\Gamma_{P}}(t)=\frac{1}{1-t^{r_{0}}} \cdot \prod_{i=1}^{h} \frac{1-t^{n_{i} r_{i}}}{1-t^{r_{i}}}=\frac{1}{1-t^{r_{0}}} \prod_{j=1}^{h} \sum_{i=0}^{n_{j}-1} t^{i r_{j}} .
$$

In particular, the Poincaré series $P_{\Gamma_{P}}(t)$ is a rational function.

Proof. The formula can be proven by considering Equation $(*)$ as follows:

$$
\begin{aligned}
& \sum_{n \in \Gamma_{P}} t^{n}=\sum_{\substack{\lambda_{0} \geq 0 \\
0 \leq \lambda_{i}<n_{i} \\
1 \leq i \leq h}} t^{\lambda_{0} r_{0}+\ldots+\lambda_{h} r_{h}} \\
& =\left(\sum_{\lambda_{0} \geq 0} t^{\lambda_{0} r_{0}}\right) \cdot\left(\sum_{0 \leq \lambda_{1}<n_{1}} t^{\lambda_{1} r_{1}}\right) \cdot \ldots \cdot\left(\sum_{0 \leq \lambda_{g}<n_{h}} t^{\lambda_{h} r_{h}}\right) \\
& =\frac{1}{1-t^{r_{0}}} \cdot \frac{1-t^{n_{1} r_{1}}}{1-t^{r_{1}}} \cdot \ldots \cdot \frac{1-t^{n_{h} r_{h}}}{1-t^{r_{h}}} .
\end{aligned}
$$

Proposition 3.3. We have

$$
P_{\Gamma_{P}}(t)=\frac{L_{\Gamma_{P}}(t)}{1-t}
$$

with $L_{\Gamma_{P}}(t)=t^{c}+(1-t) \sum_{\substack{n \in \Gamma_{P} \\ n<c}} t^{n} \in \mathbb{Z}[t]$.

Proposition 3.4. Let $X$ be a non-singular curve in the affine part, let $P$ be a closed point on $X$. The conductor of $\Gamma_{P}$ is given by

$$
c\left(\Gamma_{P}\right)=1-r_{0}+\sum_{j=1}^{h}\left(n_{j}-1\right) r_{j}
$$

therefore, the Frobenius number of $\Gamma_{P}$ is given by

$$
g\left(\Gamma_{P}\right)=\sum_{j=1}^{h}\left(n_{j}-1\right) r_{j}-r_{0}
$$


Proof. By Proposition 3.2 one has $\left(1-t^{r_{0}}\right) P_{\Gamma_{P}}(t)=\widetilde{L}_{\Gamma_{P}}(t)$, where

$$
\widetilde{L}_{\Gamma_{P}}(t)=\prod_{j=1}^{h}\left(1+t^{r_{j}}+t^{2 r_{j}}+\ldots+t^{\left(n_{j}-1\right) r_{j}}\right) .
$$

This together with Proposition 3.3 leads to the equality $(1+t+\ldots+$ $\left.t^{r_{0}-1}\right) L_{\Gamma_{P}}(t)=\widetilde{L}_{\Gamma_{P}}(t)$. By comparing the leading terms on both sides of the equality we obtain the result.

Corollary 3.5. Let $X$ be non-singular in the affine part and affine complete intersection. Then the genus of $X$ is given by the equality

$$
g_{X}=\sum_{j=1}^{h} \frac{\left(n_{j}-1\right) r_{j}}{2}+\frac{1-r_{0}}{2} .
$$

Proof. The equality is a consequence of the fact that $X$ is an affine complete intersection if and only if $c=2 g_{X}$ (cf. [16]).

Another consequence of Proposition 3.4 is a characterisation of the gaps of $\Gamma_{P}$ for an affine complete intersection curve.

Corollary 3.6. Let $X$ be non-singular in the affine part and affine complete intersection. Let $m \in \mathbb{Z}$. Then $m$ is a gap of $\Gamma_{P}$ if and only if there exist $\lambda_{0}, \ldots, \lambda_{h}>0$ such that

$$
m=\sum_{i=1}^{h} n_{i} r_{i}-\sum_{j=0}^{h} \lambda_{j} r_{j} .
$$

Proof. Assume $m \notin \Gamma_{P}$. Since the semigroup is symmetric under these hypothesis (see [16]), and by the formula for the computation of the Frobenius number of $\Gamma$ given in Proposition 3.4, we have that

$$
\sum_{j=1}^{h}\left(n_{j}-1\right) r_{j}-r_{0}-m \in \Gamma_{P} .
$$

Therefore, there exist $\alpha_{0}, \ldots, \alpha_{h} \geq 0$ such that

$$
\sum_{j=1}^{h}\left(n_{j}-1\right) r_{j}-r_{0}-m=\sum_{i=0}^{h} \alpha_{i} r_{i},
$$

and we deduce that

$$
m=\sum_{i=1}^{h} n_{i} r_{i}-\sum_{j=0}^{h}\left(\alpha_{j}+1\right) r_{j} .
$$


Conversely, suppose that $m=\sum_{i=1}^{h} n_{i} r_{i}-\sum_{j=0}^{h} \lambda_{j} r_{j}$ for $\lambda_{j}>0$ for all $0 \leq j \leq h$. Assuming that $m \in \Gamma_{P}$, then

$$
\sum_{i=1}^{h} n_{i} r_{i}-\sum_{j=0}^{h} \lambda_{j} r_{j}+\sum_{i=0}^{h}\left(\lambda_{i}-1\right) r_{i} \in \Gamma_{P},
$$

but on the other hand this is in fact equal to the Frobenius number (by Proposition 3.4), i.e., a gap of $\Gamma_{P}$, which is a contradiction.

3.2. Poincaré series associated with two points. Let $\Gamma_{P_{1}, P_{2}}$ be the Weierstraß semigroup associated with two rational points $P_{1}, P_{2}$ on $X$. Let us define the series

$$
L_{\Gamma_{P_{1}, P_{2}}}(\underline{t}):=\left(1-t_{1}\right)\left(1-t_{2}\right) P_{\Gamma_{P_{1}, P_{2}}}(\underline{t}) .
$$

This expression can be rewritten as

$$
L_{\Gamma_{P_{1}, P_{2}}}(\underline{t})=\sum_{\underline{m} \in \mathbb{Z}^{2}} c(\underline{m}) \underline{\underline{m}},
$$

where $c(\underline{m}):=d(\underline{m})-d\left(\underline{m}-e_{1}\right)-d\left(\underline{m}-e_{2}\right)+d(\underline{m}-\underline{1})$. We will describe the series $L_{\Gamma_{P_{1}, P_{2}}}(\underline{t})$ in terms of the set $\mathcal{M}_{P_{1}, P_{2}}$ of maximal points of $\Gamma_{P_{1}, P_{2}}$ in Proposition 3.8. First, a summary of the most elementary properties of the dimensions $d(\underline{m})$, for $\underline{m}=\left(m_{1}, m_{2}\right) \in \mathbb{Z}^{2}$ is presented. This can be found in Carvalho-Torres [4, Sect. 2]

Lemma 3.7. Let $\Gamma_{P_{1}, P_{2}}$ be the Weierstraß semigroup associated with two rational points $P_{1}, P_{2}$ on $X$. The following statements hold:

(1) $\underline{m} \in \Gamma_{P_{1}, P_{2}}$ if and only if $d\left(\underline{m}-e_{i}\right)=1$ for $i \in\{1,2\}$.

(2) Let $\underline{m} \in \Gamma_{P_{1}, P_{2}}$. Then $d(\underline{m})=1$ if and only if $\nabla(\underline{m})=\varnothing$.

(3) $d(\underline{m})=2$ if and only if $\underline{m} \in \Gamma_{P_{1}, P_{2}}$ and $\nabla(\underline{m}) \neq \varnothing$.

(4) If $m_{1} \in \Gamma_{P_{1}}$ and $m_{2} \in \Gamma_{P_{2}}$, then $d(\underline{m})=2$.

Proposition 3.8. Let $\underline{m} \in \mathbb{Z}^{2}$. Then we have

(1) $c(\underline{m})=-1$ if and only if $\underline{m}-\underline{1}$ is maximal.

(2) $c(\underline{m})=1$ if and only if $\underline{m}$ is maximal.

Proof. Taking into account the main properties of the Weierstraß semigroup of Lemma 3.7, and since $c(\underline{m})=d(\underline{m})-d\left(\underline{m}-e_{1}\right)-d(\underline{m}-$ $\left.e_{2}\right)+d(\underline{m}-\underline{1})$, we consider the different values the coefficients $d(\underline{m})$ can take. So, if $d(\underline{m})=2$, then the following statements hold:

- if $d\left(\underline{m}-e_{1}\right)=d\left(\underline{m}-e_{2}\right)=1$, then the only possible case is $d(\underline{m}-\underline{1})=0$ and $c(\underline{m})=2-1-1+0=0$;

- if $d\left(\underline{m}-e_{1}\right)=1$ and $d\left(\underline{m}-e_{2}\right)=2$, then $d(\underline{m}-\underline{1})=1$ and from this $c(\underline{m})=2-1-2+1=0$; 
- if $d\left(\underline{m}-e_{1}\right)=2$ and $d\left(\underline{m}-e_{2}\right)=1$, as in the previous case one has $d(\underline{m}-\underline{1})=1$ and $c(\underline{m})=2-2-1+1=0$;

- if $d\left(\underline{m}-e_{1}\right)=d\left(\underline{m}-e_{2}\right)=2$, one has $d(\underline{m}-\underline{1}) \geq 1$. If $d(\underline{m}-\underline{1})=2$, then $c(\underline{m})=2-2-2+2=0$; on the other hand, if $d(\underline{m}-\underline{1})=1$, then $c(\underline{m})=-1$ and $\nabla(\underline{m}-\underline{1})=\varnothing$ by Lemma 3.7 (2). Thus the point $\underline{m}-\underline{1}$ is maximal.

In the case $d(\underline{m})=1$, we have to distinguish whether the point $\underline{m}$ is maximal. If this is the case,

- if $d\left(\underline{m}-e_{1}\right)=d\left(\underline{m}-e_{2}\right)=1$, one has either (i) $d(\underline{m}-\underline{1})=1$ implying that $c(\underline{m})=1-1-1+1=0$ and $\underline{m}-\underline{1}$ is maximal; or (ii) $d(\underline{m}-\underline{1})=2$ with $c(\underline{m})=1-1-1+2=1$, hence $\underline{m}-\underline{1}$ is not maximal by Lemma 3.7(3);

- if $d\left(\underline{m}-e_{1}\right)=1$ and $d\left(\underline{m}-e_{2}\right)=0$, then one has only $d(\underline{m}-\underline{1})=$ 1 and $c(\underline{m})=1-1-0+1=1$, hence $\underline{m}-\underline{1}$ cannot be maximal;

- if $d\left(\underline{m}-e_{1}\right)=0$ and $d\left(\underline{m}-e_{2}\right)=1$, then one has again $d(\underline{m}-\underline{1})=$ 1 and $c(\underline{m})=1$, where again $\underline{m}-\underline{1}$ has to be non-maximal;

- if $d\left(\underline{m}-e_{1}\right)=0=d\left(\underline{m}-e_{2}\right)=0$, then $d(\underline{m}-\underline{1})=0$ and $c(\underline{m})=1$.

On the other hand, if $\underline{m}$ is non-maximal, it holds that

- if $d\left(\underline{m}-e_{1}\right)=0$ and $d\left(\underline{m}-e_{2}\right)=1$, then $d(\underline{m}-\underline{1})=0$ and the dimension $c(\underline{m})$ has to be equal to $c(\underline{m})=1-0-1+0=0$.

- if $d\left(\underline{m}-e_{1}\right)=0$ and $d\left(\underline{m}-e_{2}\right)=2$, then we have only $d(\underline{m}-\underline{1})=$ 1 and $c(\underline{m})=1-0-2+1=0$;

- if $d\left(\underline{m}-e_{1}\right)=1=d\left(\underline{m}-e_{2}\right)=1$, then again $d(\underline{m}-\underline{1})=1$ and analogously $c(\underline{m})=1-1-1+1=0$;

- if $d\left(\underline{m}-e_{1}\right)=1$ and $d\left(\underline{m}-e_{2}\right)=2$, then one has either (i) $d(\underline{m}-\underline{1})=1$, therefore $c(\underline{m})=1-1-2+1=-1$ and $\underline{m}-\underline{1}$ has to be maximal by Lemma $3.7(2)$; or (ii) $d(\underline{m}-\underline{1})=2$, hence $c(\underline{m})=1-1-2+2=0$.

The remaining case is $d(\underline{m})=0$, which is investigated in a similar manner:

- if $d\left(\underline{m}-e_{1}\right)=d\left(\underline{m}-e_{2}\right)=0$, then $d(\underline{m}-\underline{1})=0$ and $c(\underline{m})=0$;

- if $d\left(\underline{m}-e_{1}\right)=0$ and $d\left(\underline{m}-e_{2}\right)=1$, then $d(\underline{m}-\underline{1})=0$ and $c(\underline{m})=0$

- if $d\left(\underline{m}-e_{1}\right)=1$ and $d\left(\underline{m}-e_{2}\right)=0$ one has again $d(\underline{m}-\underline{1})=0$ and $c(\underline{m})=0$;

- if $d\left(\underline{m}-e_{1}\right)=d\left(\underline{m}-e_{2}\right)=1$, then $d(\underline{m}-\underline{1})=2$ and $c(\underline{m})=0$.

This finishes the proof. 


\section{Corollary 3.9.}

$$
L_{\Gamma_{P_{1}, P_{2}}}(\underline{t})=\left(1-t_{1} \cdot t_{2}\right) \sum_{\underline{m} \in \mathcal{M}_{P_{1}, P_{2}}} \underline{t} \underline{\underline{m}} .
$$

Proof. The basic idea of the proof is to observe

$$
\begin{aligned}
L_{\Gamma_{P_{1}, P_{2}}}(\underline{t}) & =\sum_{\underline{m} \in \mathcal{M}_{P_{1}, P_{2}}} \underline{t} \underline{\underline{m}}+\sum_{\underline{m}-\underline{1} \in \mathcal{M}_{P_{1}, P_{2}}} \underline{t} \underline{\underline{m}} \\
& =\sum_{\underline{m} \in \mathcal{M}_{P_{1}, P_{2}}} \underline{t} \underline{\underline{m}}-\sum_{\underline{m} \in \mathcal{M}_{P_{1}, P_{2}}} \underline{t}^{\underline{m}+1} .
\end{aligned}
$$

Theorem 3.10. Let $\mathcal{C}:=\mathcal{C}_{P_{1}, P_{2}}$ be the fundamental corner of the semigroup $\Gamma_{P_{1}, P_{2}}$ and consider the set $\mathcal{M}_{\mathcal{C}}:=\mathcal{M}_{P_{1}, P_{2}} \cap \mathcal{C}$. Then we have

$$
L_{\Gamma_{P_{1}, P_{2}}}(\underline{t})=\left(1-t_{1} \cdot t_{2}\right) \sum_{\underline{m} \in \mathcal{M}_{\mathcal{C}}} \underline{t} \underline{\underline{m}} .
$$

Proof. We denote $\underline{\vartheta}^{\prime}:=(\vartheta,-\vartheta)$. The series $L_{\Gamma_{P_{1}, P_{2}}}(\underline{t})$ can be written as

$$
\begin{aligned}
& L_{\Gamma_{P_{1}, P_{2}}}(\underline{t})=\left(1-t_{1} \cdot t_{2}\right) \cdot \sum_{\underline{m} \in \mathcal{M}_{P_{1}, P_{2}}} \underline{t}^{\underline{m}}
\end{aligned}
$$

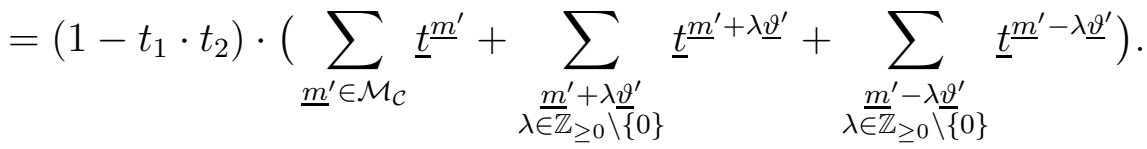

The proof is completed by showing that the latter two summands are 0 .

Corollary 3.11. We have

$$
\begin{aligned}
P_{\Gamma_{P_{1}, P_{2}}}(\underline{t}) & =\frac{L_{\Gamma_{P_{1}, P_{2}}}(\underline{t})}{\left(1-t_{1}\right)\left(1-t_{2}\right)} \\
& =\frac{1-t_{1} t_{2}}{\left(1-t_{1}\right)\left(1-t_{2}\right)} \sum_{\underline{m} \in \mathcal{M}_{\mathcal{C}}} \underline{t} \underline{\underline{m}} .
\end{aligned}
$$

\section{Symmetry AND FUnCTIONAL EQUATIONS}

\subsection{The symmetry of the semigroup and affine embeddings.}

Some important considerations about the symmetry of the Weierstraß semigroup at several points were introduced by Delgado in [7], being the most remarkable the following result. 
Theorem 4.1 (Delgado). Let $X$ be a reducible projective space curve of arithmetical genus $g_{X}$ and $X^{\prime}=X \backslash\left\{P_{1}, \ldots, P_{r}\right\}$, where $P_{i}$ are smooth points for all $i \in\{1, \ldots, r\}$. Then, the following statements are equivalent:

(1) $X^{\prime}$ is an affine complete intersection.

(2) There exists a canonical divisor $K$ such that

$$
\operatorname{supp}(K) \subset\left\{P_{1}, \ldots, P_{r}\right\} .
$$

(3) There exists a relative maximal $\underline{\tau}=\left(\tau_{1}, \ldots, \tau_{r}\right)$ in the semigroup $\Gamma_{\underline{P}}$ such that $\sum_{i=1}^{r} \tau_{i}=2 g_{X}-2+r$.

(4) There exists $\underline{\sigma}=\left(\sigma_{1}, \ldots, \sigma_{r}\right) \in \Gamma_{\underline{P}}$ such that $\sum_{i=1}^{r} \sigma_{i}=2 g_{X}-$ $2+r$ and $\Gamma_{\underline{P}}$ is symmetric with respect to $\underline{\sigma}$.

Proof. The equivalences $(2) \Leftrightarrow(3) \Leftrightarrow(4)$ go straight as in [7]. The rest of the subsection is devoted to present the suitable results to reach the equivalence $(1) \Leftrightarrow(2)$.

The rest of the Subsection is devoted to present in a self-contained way the proof " $(1) \Leftrightarrow(2)$ " of Theorem 4.1. (A clear ordering in the steps is missing in the proof of Delgado [7, p. 630]). First, we collect now some known results due to Serre (cf. [17]).

Lemma 4.2. Let $R$ be a ring. Assume that every projective $R$-module of ranks 1 and 2 are free. Let $I$ be a nonzero ideal of $R$ with projective dimension less than or equal to 1 . The following statements are equivalent:

(1) I may be generated by two elements.

(2) $\operatorname{Ext}_{R}^{1}(I, R)$ is a cyclic $R$-module.

Proof. See [17, §2.4., Corollaire].

Let $V$ be a non-singular variety of dimension $r, \mathcal{O}_{V}$ be the affine coordinate ring of $V$ and $\Omega_{V}$ be the sheaf of differential forms of degree $r$ over $V$. It is a locally free sheaf of rank 1 . Let $W$ be a subvariety of $V$ of codimension $h$.

Definition 4.3. The module of differential forms on $W$ is defined to be

$$
\Omega_{W}:=\operatorname{Ext}_{\mathcal{O}_{V}}^{h}\left(\mathcal{O}_{W}, \Omega_{V}\right)
$$

Remark 4.4. For any ring $R$, there is a correspondence between free finitely generated projective $R$-modules of finite rank and trivial vector bundles on $\operatorname{Spec}(R)$ of the same rank.

The next result was also proven by Serre (cf. [17, §2.7., Prop. 6]). By the sack of completeness, we rewrite his proof, adding further details. 
Proposition 4.5. Let $V$ be a non-singular affine variety over which every vector bundle of rank 1 is trivial. Let $W \subset V$ be a CohenMacaulay subvariety of $V$ of codimension 2. Then, if $W$ is a complete intersection on $V$, then $\Omega_{W} \cong \mathcal{O}_{W}$. Conversely, assuming moreover that every vector bundle of rank 2 on $V$ is trivial, we have that $W$ is a complete intersection on $V$ if $\Omega_{W} \cong \mathcal{O}_{W}$.

Proof. The sheaf $\Omega_{V}$ is locally free of rank 1. Every algebraic vector bundle of rank 1 is trivial by hypothesis, then $\Omega_{V} \cong \mathcal{O}_{V}$. Furthermore, if $W$ is a complete intersection, we have the exact sequence

$$
0 \rightarrow \mathcal{A}_{W} \rightarrow \mathcal{O}_{V} \rightarrow \mathcal{O}_{W} \rightarrow 0
$$

where $\mathcal{A}_{W}$ denotes the coherent ideal sheaf defining $W$. By applying the functor $\operatorname{Ext}_{\mathcal{O}_{V}}\left(-, \mathcal{O}_{V}\right)$ to this exact sequence, the necessity is proven. Conversely, let $\mathcal{O}_{V}$ be the affine coordinate ring of $V$, and $\mathcal{A}_{W}$ the ideal defining $W$. Then the sheaf associated to $\operatorname{Ext}_{\mathcal{O}_{V}}^{2}\left(\mathcal{O}_{W}, \mathcal{O}_{V}\right) \cong$ $\operatorname{Ext}_{\mathcal{O}_{V}}^{2}\left(\mathcal{O}_{W}, \Omega_{V}\right)$ is isomorphic to $\Omega_{W}$, and $\Omega_{W} \cong \mathcal{O}_{W}$, thus

$$
\operatorname{Ext}_{\mathcal{O}_{V}}^{2}\left(\mathcal{O}_{W}, \mathcal{O}_{V}\right) \cong \mathcal{O}_{W}
$$

and so it is cyclic. On the other hand, since $\mathcal{O}_{W}$ is locally CohenMacaulay, the homological dimension of the ideal defining $W$ is at most 1. Remark 4.4 allows us to apply Lemma 4.2 and see that this ideal may be generated by two elements, i.e., $W$ is a complete intersection on $V$.

Remark 4.6. Let $R$ be a principal ideal domain. Then every finitely generated projective $R\left[x_{1}, \ldots, x_{n}\right]$-module is free (see [14, Theorem 4, p. 169]).

Finally, we reach the statement $(1) \Leftrightarrow(2)$ of Theorem 4.1 .

Corollary 4.7. Every non-singular affine curve is a complete intersection if and only if there exists a canonical divisor with support totally contained in the places at infinity of the curve.

Proof. The existence of a canonical divisor totally supported in places at infinity for the curve is equivalent to the existence of a trivial canonical line bundle. Let $\mathbb{A}^{n}=\operatorname{Spec}\left(\mathbb{F}\left[x_{1}, \ldots, x_{n}\right]\right)$. Since $\mathbb{F}$ is a field, every finitely generated projective module over $\mathbb{F}\left[x_{1}, \ldots, x_{n}\right]$ is free by Remark 4.6, and that means by Remark 4.4 that every vector bundle on $\operatorname{Spec}\left(\mathbb{F}\left[x_{1}, \ldots, x_{n}\right]\right)=\mathbb{A}^{n}$ is trivial. Let $W$ be a smooth affine curve, then it is Cohen-Macaulay. Now, from Proposition 4.5, taking $V=\mathbb{A}^{n}$ and $W$ the smooth affine curve, that is the case if and only if $W$ is a complete intersection. 
4.2. Functional equations. Poincaré series associated with value semigroups of curve singularities, or zeta functions associated with local singular rings, satisfy functional equations whenever the base ring is Gorenstein (see [6] and [18]; also [12]). The same holds in our global context for complete intersections.

Definition 4.8. The point $\underline{\sigma}$ of the last assertion of Theorem 4.1 is called the symmetry point of $\Gamma_{\underline{P}}$.

For the case $r=1$, the symmetry point of $\Gamma_{P}$ is the conductor, which is equal to $c\left(\Gamma_{P}\right)=2 g_{X}$ (cf. [16]). Then we can deduce easily the functional equations.

Lemma 4.9. Let $X$ be a curve. Let $P$ be a rational point of $X$ such that $X \backslash P$ is an affine complete intersection. We have

$$
\begin{aligned}
& L_{\Gamma_{P}}(t)=-t^{2 g_{X}} L_{\Gamma_{P}}\left(t^{-1}\right), \\
& P_{\Gamma_{P}}(t)=t^{2 g_{X}-1} P_{\Gamma_{P}}\left(t^{-1}\right) .
\end{aligned}
$$

Proof. Let $n \in \mathbb{Z}$. By the definition of conductor of a numerical semigroup, we know that $n \in \Gamma_{P}$ if and only if $2 g_{X}-1-n \notin \Gamma_{P}$; it implies that $d\left(2 g_{X}-1-n\right)=0$ if and only if $d(n)=1$. It is easily checked that

$$
t^{2 g_{X}-1} P_{\Gamma_{P}}\left(t^{-1}\right)=\sum_{m \in \mathbb{Z}} d(m) t^{2 g_{X}-1-n}=\sum_{n \in \Gamma_{P}} t^{n}=P_{\Gamma_{P}}(t) .
$$

The functional equation for $L_{\Gamma_{P}}(t)$ follows from a simple computation.

The next result establishes the functional equation for the Poincaré series associated with two points.

Proposition 4.10. Let $X$ be a curve, let $P_{1}, P_{2}$ be two rational points on $X$ such that $X \backslash\left\{P_{1}, P_{2}\right\}$ is an affine complete intersection. Let us denote by $\underline{\sigma}$ the symmetry point of the Weierstraß semigroup $\Gamma_{P_{1}, P_{2}}$. We have

$$
\begin{aligned}
& L_{\Gamma_{P_{1}, P_{2}}}(\underline{t})=-\underline{t}^{\underline{\sigma}+\underline{1}} \cdot L_{\Gamma_{P_{1}, P_{2}}}\left(\underline{t}^{-1}\right), \\
& P_{\Gamma_{P_{1}, P_{2}}}(\underline{t})=-\underline{t}^{\underline{\sigma}} \cdot P_{\Gamma_{P_{1}, P_{2}}}\left(\underline{t}^{-1}\right) .
\end{aligned}
$$

Proof. By Proposition 4.5, we have

$$
L_{\Gamma_{P_{1}, P_{2}}}(\underline{t})=\left(1-t_{1} \cdot t_{2}\right) \cdot \sum_{\underline{m} \in \mathcal{M}_{P_{1}, P_{2}}} \underline{t} \underline{\underline{m}} .
$$


Moreover, for a maximal point $\underline{m} \in \mathcal{M}_{P_{1}, P_{2}}$, there exists $\underline{n} \in \mathcal{M}_{P_{1}, P_{2}}$ such that $\underline{n}+\underline{m}=\underline{\sigma}$. Hence

$$
\begin{aligned}
L_{\Gamma_{P_{1}, P_{2}}}(\underline{t}) & =\left(1-t_{1} \cdot t_{2}\right) \cdot \sum_{\underline{m} \in \mathcal{M}_{P_{1}, P_{2}}} \underline{t}^{\underline{m}} \\
& =\left(1-t_{1} \cdot t_{2}\right) \cdot \sum_{\underline{n} \in \mathcal{M}_{P_{1}, P_{2}}} \underline{t}^{\underline{\sigma}-\underline{n}} \\
& =-\underline{t}^{\underline{\sigma}+\underline{1}} \cdot L\left(\underline{t}^{-1}\right) .
\end{aligned}
$$

On the other hand, by the definition and the functional equation of $L_{\Gamma_{P_{1}, P_{2}}}(\underline{t})$, we have

$$
\begin{aligned}
P_{\Gamma_{P_{1}, P_{2}}}\left(\underline{t}^{-1}\right) & =\frac{L_{\Gamma_{P_{1}, P_{2}}}\left(\underline{t}^{-1}\right)}{\left(1-t_{1}^{-1}\right)\left(1-t_{2}^{-1}\right)} \\
& =\frac{-\underline{t}^{-\underline{\sigma}} \cdot L_{\Gamma_{P_{1}, P_{2}}}(\underline{t})}{\left(1-t_{1}\right)\left(1-t_{2}\right)} \\
& =-\underline{t}^{-\underline{\sigma}} \cdot P_{\Gamma_{P_{1}, P_{2}}}(\underline{t}) .
\end{aligned}
$$

\section{ACKNOWLEDGEMENTS}

The author is thankful to Professor Félix Delgado de la Mata for stimulating conversations on the combinatorial structure of value semigroups, as well as to the referee of this paper for his/her helpful comments.

\section{REFERENCES}

[1] S. S. Abhyankar: Lectures on expansion techniques in Algebraic Geometry. Tata Institute of Fundamental Research, Bombay, 1977.

[2] A. Brill, M. Noether: Über die algebraischen Functionen und ihre Anwendung in der Geometrie. Math. Ann. 7 (1874), 269-310.

[3] A. Campillo, F. Delgado, S. M. Gusein-Zade: On the monodromy at infinity of a plane curve and the Poincaré series of its coordinate ring. Vol. in honour to Pontryagin. Conteporary Math. and its Applications, Moscow (1998). English version: J. Math. Sciences (New York), 105(2) (2001), 1839-1842.

[4] C. Carvalho, F. Torres: On Goppa codes and Weierstrass gaps at several points. Designs, Codes and Cryptography 35(2) (2005), 211-225.

[5] A. Del Centina: Weierstrass points and their impact in the study of algebraic curves: a historical account from the "Lückensatz" to the 1970s. Ann. Univ. Ferrara 54 (2008), 37-59.

[6] F. Delgado: Gorenstein curves and symmetry of the semigroup of values. Manuscripta math. 61 (1988), 285-296.

[7] F. Delgado: The symmetry of the Weierstrass generalized semigroups and affine embeddings. Proc. Amer. Math. Soc. 108(3) (1990), 627-631. 
[8] J. Herzog, E. Kunz: Die Wertehalbgruppe eines lokalen Rings der Dimension 1. Ber. Heidelberger Akad. Wiss. 1971, II. Abh. (1971), 27-67.

[9] A. Hurwitz: Über algebraische Gebilde mit eindeutigen Transformationen in sich. Math. Ann. 41 (1893), 403-442.

[10] K. Kiyek, J. L. Vicente: Resolution of Curve and Surface Singularities in Characteristic Zero. Kluwer, Dordrecht, 2004.

[11] Q. Liu: Algebraic Geometry and Arithmetic Curves. Oxford U.P., Oxford, 2002.

[12] J.J. Moyano-Fernández, W.A. Zúñiga Galindo: Motivic zeta functions for curve singularities. Nagoya Math. Journal 198, 47-75 (2010).

[13] F. Oort: Reducible and multiple algebraic curves. Van Gorcum \& Comp. N.V., Assen, 1961.

[14] D. Quillen: Projective modules over polynomial rings. Inventiones math. 36 (1976), 167-171.

[15] J.C. Rosales, P.A. Garcia Sanchez: Numerical semigroups. Developments in Mathematics, Vol. 20. Springer, Berlin-Heidelberg-New York, 2010.

[16] A. Sathaye: On planar curves. American Journal of Math. 99 (1977), 11051135 .

[17] J. P. Serre: Sur les modules projectivs. Séminaire Dubreil-Pisot 1 (1960/61).

[18] K. O. Stöhr: Local and Global Zeta-Functions of Singular Algebraic Curves. Journal of Number Theory $\mathbf{7 1}$ (1998), 172-202.

[19] K. Weierstraß: Mathematische Werke. Berlin (1894-1927). Reprinted by G. Olms, Hildesheim, 1967.

Institut für Mathematik, Universität Osnabrück. Albrechtstrasse 28A, D-49076 Osnabrück, Germany

E-mail address: jmoyanof@uni-osnabrueck.de 